\title{
Sodium valproate in the treatment of levodopa-induced dyskinesia
}

\author{
P.A.PR I CE, J. D. PARKES, A N D C. D. MAR S D N \\ From the University Department of Neurology, Institute of Psychiatry, and King's College Hospital, \\ London
}

SUMMARY The effect of sodium valproate $1200 \mathrm{mg}$ daily on the disability of Parkinsonism and on levodopa-induced dyskinesias was assessed in a double-blind crossover trial with matched placebo in 12 patients with Parkinson's disease. No objective change in the severity of Parkinsonism or dyskinesias was noted. However, six out of nine patients who completed the trial noted a slight to moderate improvement in their dyskinesias with no change in their Parkinsonism. Excess salivation improved in four subjects on sodium valproate.

The amino acid gamma-aminobutyric acid (GABA) is widespread throughout the nervous system where it generally functions as an inhibitory neurotransmitter (Roberts, 1974). The importance of GABA in the motor system of man is not known, although the highest concentration of GABA in animals and man is found in the basal ganglia (Bird, 1976). The concentration of GABA and its synthesising enzyme 1-glutamic acid decarboxylase is reduced by half in patients dying of Parkinson's disease (McGeer et al., 1973).

In mammals, there is a GABAminergic pathway descending from the neostriatum to the substantia nigra (Kim et al., 1971) which mediates an inhibitory effect on ascending nigrostriatal dopaminergic pathways (Tarsy et al., 1975). Thus, manipulation of GABA levels in the basal ganglia might be useful for the treatment of Parkinson's disease or affect the side effects of antiparkinsonism medication.

Linnoila et al. (1976) have reported that sodium valproate (Epilim, Reckitt-Labaz), which raises brain GABA levels, improves the features of chronic tardive dyskinesias. We have investigated the action of sodium valproate in people with Parkinson's disease to determine whether this drug alters any aspect of disability in this disorder as well as to determine the action on levodopainduced dyskinesias.

Address for reprint requests : Dr J. D. Parkes, King's College Hospital, London SE5 9RS.

Accepted 18 February 1978

\section{Patients and methods}

Twelve patients with idiopathic Parkinson's disease (seven men and five women; five mildly disabled and seven moderately disabled; mean age 64.3 years) were selected for study. Mean duration of disease was 11 years, and all patients displayed troublesome involuntary movements on levodopa. Three patients had orofacial dyskinesias, two had choreic or dystonic movements of the limbs or trunk or both, and seven had both orofacial dyskinesias and limb-trunk movements.

\section{DRUGS}

Four patients were taking levodopa alone (mean daily dose $2.2 \mathrm{~g}$ ) and eight were taking levodopa (mean daily dose $0.59 \mathrm{~g}$ ) combined with 1-alphamethyldopahydrazine, an extracerebral dopa decarboxylase inhibitor. One patient was taking bromocriptine $100 \mathrm{mg}$ per day in addition to levodopa. Dosages of levodopa, bromocriptine, amantadine (10 patients), anticholinergic drugs (seven patients), or metoclopramide (two patients) were not changed during the trial.

PROTOCOL

Subjects were treated as outpatients with sodium valproate (200 $\mathrm{mg}$ tablets) and matched placebo for six weeks each, in a crossover sequence. The order of drugs taken was allocated randomly by an independent person. Patients and the examiner were unaware of the treatment order used in each case until after completion of the trial. An identical regime of drug or placebo given in 
increasing doses was used. On weeks 1 and 7 one tablet daily, on weeks 2 and 8 one tablet twice daily, on weeks 3 and 9 one tablet thrice daily, on weeks 4 and 10 two tablets twice daily, and on weeks $5,6,11$, and 12 two tablets thrice daily, were given.

Every fortnight, patients returned unused tablets to the pharmacy so that an estimate of patient compliance could be made.

\section{ASSESSMENT}

Patients were assessed at the beginning of the trial and at intervals of two weeks thereafter for 12 weeks by the same observer. Assessments for total disability, tremor, rigidity, akinesia, and postural flexion were made (Marsden et al., 1973). Dyskinesias were scored on a scale of $0-3,0$ being no dyskinesias, 3 being very severe dyskinesias. Both the nature (for example, chorea or dystonia) and the localisation of dyskinesias (for example, mouth, hand, foot) were determined separately and these separate scores were added.

On each attendance, patients were asked to comment separately on whether orofacial dyskinesias and limb-trunk dyskinesias were better, unchanged, or worse as compared with the previous attendance. The patients' own assessment of their disability from Parkinsonism and the occurrence of any unwanted effects was also noted.

Blood count, ESR, plasma urea and electrolytes, liver function tests, serum proteins, and serum calcium and phosphate were determined on each patient at the beginning and end of the trial. No alteration in these measurements occurred during sodium valproate treatment.

Three patients failed to complete the trial, one due to hospitalisation for carcinoma of the bronchus, one because of an inability to adhere to a constant drug regime, and one because of intolerable nausea and depression on placebo.

\section{Results}

Seven out of nine patients completed both active and placebo phases of the trial. Two subjects (patients 3 and 5 in the Table) curtailed the placebo phase (given first in each case) because of worsening of orofacial dyskinesia in one case and worsening of hallucinations and limb chorea in the other. These two patients completed the active drug phase of the trial and have been included in the analysis.

EFFECT OF SODIUM VALPROATE ON DISABILITY OF PARKINSONISM

Sodium valproate did not have any objective effect on disability of Parkinsonism. Mean total Parkinsonism disability scores before treatment, after placebo, and after sodium valproate, were not significantly different (Table; $P>0.05$ in each case). Subscores for tremor, rigidity, akinesia, postural flexion, and functional disability were also not significantly different at the beginning of the trial, after placebo, or after sodium valproate. There was a marked variation in Parkinsonism disability score and subscores in some but not all patients on different occasions on a constant treatment regime.

EFFECT OF SODIUM VALPROATE ON LEVODOPAINDUCED DYSKINESIAS

The severity of levodopa-induced dyskinesias was not objectively altered by sodium valproate. Mean total dyskinesia scores before treatment, after placebo, and after sodium valproate were not significantly different ( $P>0.05$ in each case). Those patients who showed a marked variation in Parkinsonism disability scores on successive clinic attendances also had a considerable difference in total dyskinesia scores on different dates.

PATIENTS' SUBJECTIVE RESPONSES

Seven out of nine patients felt that their Parkinsonism disability was unchanged on sodium valproate as compared with placebo. Patient 8 noted a very slight deterioration in Parkinsonism disability as compared with placebo, patient 5 (who did not complete the placebo phase of the trial) noted a deterioration in walking with sodium valproate $1200 \mathrm{mg}$ daily, but not with lower dosages. In this patient, there was a subjective improvement in his dyskinesia at dosages below $1200 \mathrm{mg}$.

Six out of nine patients noted a subjective improvement in their dyskinesias on sodium valproate compared with placebo and also compared with the beginning of the trial. Of these, two patients remarked on a moderate improvement in dyskinesias and four patients noted a slight improvement. One patient noted a slight worsening of dyskinesias on sodium valproate, with placebo having no effect. Two patients noted no alteration in their dyskinesias on either placebo or sodium valproate. Subjective improvement occurred in both orofacial dyskinesia, and limb and trunk dyskinesia. Five out of six patients noted improvement in orofacial dyskinesia on sodium valproate while three out of six patients noted improvement in limb and trunk dyskinesia on sodium valproate.

SIDE EFFECTS

No serious side effects occurred on sodium val- 
ปั้

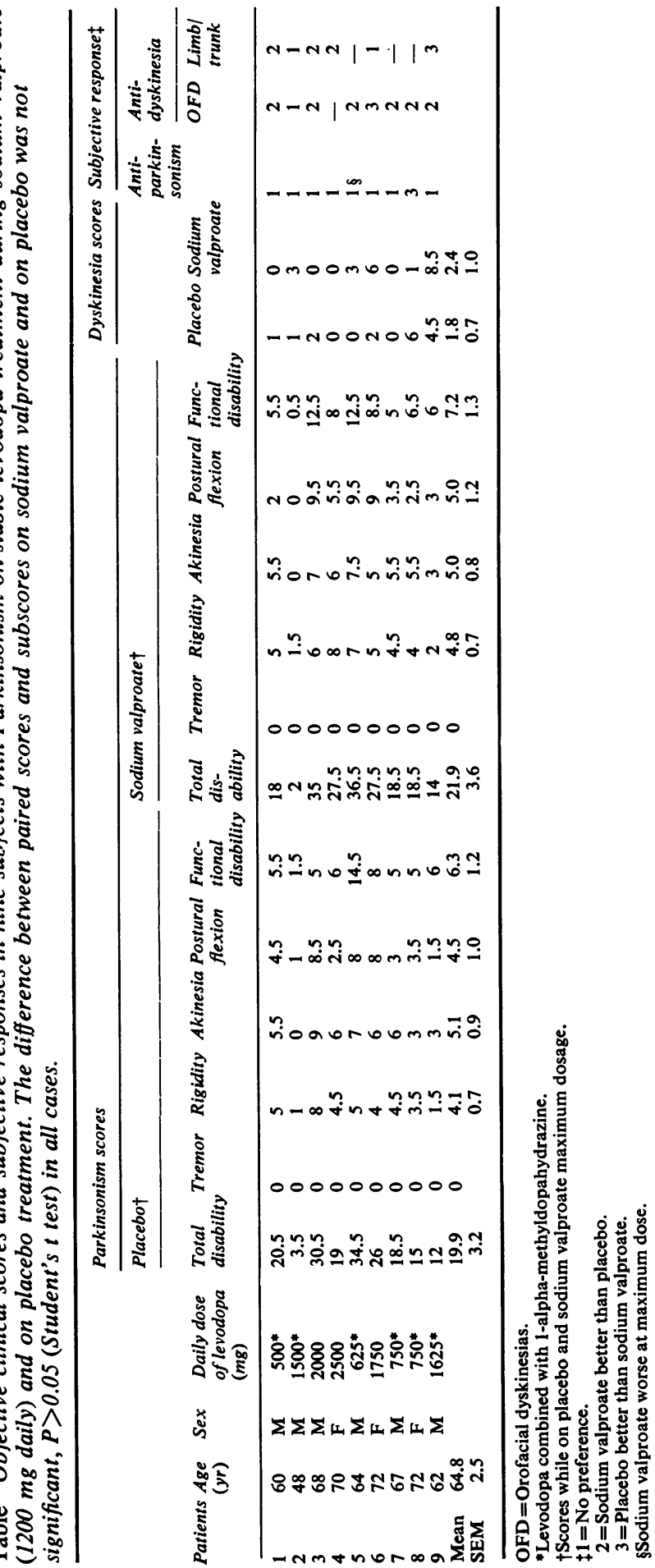


proate. Of nine patients who completed the trial, four noted a welcome decrease in their excess salivation on sodium valproate as compared to one patient on placebo. Three individual patients reported vivid dreaming, hallucinations, or drowsiness with depression on placebo, but not on sodium valproate.

DRUG ORDER AND PATIENT COMPLIANCE

Mean total Parkinsonism disability score $(21.4 \pm$ 3.2) after the first six weeks of the trial, irrespective of whether the initial treatment was active drug or placebo, was not significantly different from that after the last six weeks of the trial (20.4 $\pm 3.6 ; \quad P>0.05)$. Similarly, total dyskinesia scores were not significantly different at the end of the first and second halves of the trial (2.1士 1.0 and $2.2 \pm 0.7$ respectively).

Of nine patients who completed the trial, four out of four noted a subjective improvement in dyskinesias on sodium valproate when the active drug was given first.

Compliance on sodium valproate was good (greater than $85 \%$ ) in eight out of nine patients who completed the trial. Patient 5 took only $35 \%$ of the active drug, and did not complete the placebo phase of the trial.

\section{Discussion}

These results suggest that sodium valproate may have a very minor beneficial effect in the treatment of levodopa-induced dyskinesias. With sodium valproate, the majority of subjects reported subjective improvement in dyskinesias without worsening of Parkinsonism. Although there was no objective improvement in dyskinesias, there was a considerable variation in their severity in individual patients from day to day on a constant drug regime.

The significance of striatal gamma-aminobutyric acid (GABA) loss in patients with Parkinson's disease is uncertain. GABA deficiency may be the biochemical substrate for tremor, while Barbeau (1973) has suggested that GABA deficiency may be responsible for rigidity rather than other features in Parkinsonism. Striatal GABA deficiency in Parkinson's disease may, however, be secondary to loss of dopaminergic neurones, since GABA levels return to normal after chronic levodopa therapy (Lloyd and Hornykiewicz, 1973).

The main striatal-pallidal outflow pathway is probably GABAminergic (Hattori et al., 1973) and in addition, both nigrostriatal and mesolimbic dopaminergic pathways are under inhibitory
GABAminergic control (Tarsy et al., 1975; Pycock and Horton, 1976). Elevation of pallidal and nucleus accumbens GABA levels in animals produces akinesia (Pycock et al., 1976) and diminished motor hyperactivity in response to dopaminergic drugs (Pycock and Horton, 1976). Consequently, an elevation of cerebral GABA levels might be expected to worsen some features of Parkinsonism in humans. GABAminergic drugs appear to possess a greater effect on dopamine turnover in the mesolimbic system than in the nigrostriatal system (Fuxe et al., 1975). Mesolimbic and nigrostriatal dopaminergic pathways appear to subserve different aspects of motor behaviour (Kelly and Moore, 1976). Different populations of dopamine receptors (Cools and van Rossum, 1976) at these two sites may separately mediate increased mobility in Parkinson's disease and dyskinesias after levodopa (Parkes et al., 1976). If GABAminergic drugs have a different effect on dopaminergic activity at these two sites, this may explain their ability to reduce dyskinesias but not the antiparkinsonism effect after levodopa.

It seems probable that any antidyskinetic effect of sodium valproate is related to an increase of cerebral GABA levels caused by this compound. Sodium valproate has a structure similar to GABA and in high concentrations does raise cerebral GABA levels (Anlezark et al., 1976). The effect of more potent drugs acting on GABA systems in Parkinson's disease remains to be determined.

P. A. Price is a fellow of the Parkinson's Disease Society. We gratefully acknowledge financial assistance from Reckitt and Colman. Mrs Lavinia Ganley and Miss Jane Young of King's College Hospital Pharmacy Department provided invaluable assistance.

\section{References}

Anlezark, G., Horton, R. W., Meldrum, B. S., and Sawaya, M. C. B. (1976). Anticonvulsant action of ethanolamine-O-sulphate and di-n-propylacetate and the metabolism of gamma-aminobutyric acid (GABA) in mice with audiogenic seizures. Biochemical Pharmacology, 25, 415-417.

Barbeau, A. (1973). GABA and Huntington's chorea. Lancet, 2, 1499-1500.

Bird, E. D. (1976). Biochemical studies of gammaaminobutyric acid metabolism in Huntington's chorea. In Biochemistry and Neurology, pp. 83-92. Edited by H. F. Bradford and C. D. Marsden. Academic Press: London.

Cools, A. R., and van Rossum, J. M. (1976). Excitation-mediating and inhibition-mediating dopamine receptors: a new concept towards a better 
understanding of electrophysiological, biochemical, pharmacological, functional and clinical data. Psychopharmacologia, 45, 243-254.

Fuxe, K., Hökfelt, T., Ljungdahl, A., Agnati, L., Johansson, O., and Perez de la Mora, M. (1975). Evidence for an inhibitory gabergic control of the mesolimbic dopamine neurones. Possibility of improving treatment of schizophrenia by combined treatment with neuroleptics and gabergic drugs. Medical Biology, 53, 177-183.

Hattori, T., McGeer, P. L., Fibiger, H. C., and McGeer, E. G. (1973). On the source of GABAcontaining terminals in the substantia nigra. Electron microscopic, autoradiographic and biochemical studies. Brain Research, 54, 103-114.

Kelly, P. H., and Moore, K. E. (1976). Mesolimbic dopaminergic neurones in the rotational model of nigrostriatal function. Nature, 263, 695-696.

Kim, J. S., Bak, I. J., Hassler, R., and Okoda, Y. (1971). Role of gamma-aminobutyric acid in the extrapyramidal motor system. Some evidence for the existence of GABA-rich strionigral neurones. Experimental Brain Research, 14, 95-104.

Linnoila, M., Viukari, M., and Hietala, O. (1976). Effect of sodium valproate on tardive dyskinesia. British Journal of Psychiatry, 129, 114-119.

Lloyd, K. G., and Hornykiewicz, O. (1973). LGlutamic acid decarboxylase in Parkinson's disease: effect of L-dopa therapy. Nature, 243, 521-523.

Marsden, C. D., Parkes, J. D., and Rees, J. E. (1973). A year's comparison of treatment of patients with Parkinson's disease with levodopa combined with carbidopa versus treatment with levodopa alone. Lancet, 2, 1459-1462.

McGeer, P. L., McGeer, E. G., and Fibiger, H. C. (1973). Glutamic acid decarboxylase and choline acetylase in Huntington's chorea and Parkinson's disease. Lancet, 2, 623-624.

Parkes, J. D., Bedard, P., and Marsden, C. D. (1976). Chorea and torsion in Parkinsonism. Lancet, 2, 155.

Pycock, C. J., and Horton, R. W. (1976). Possible GABA-mediated control of dopamine-dependent behavioural effects from the nucleus accumbens of the rat. Psychopharmacology, 49, 173-178.

Pycock, C. J., Horton, R. W., and Marsden, C. D. (1976). The behavioural effects of manipulating GABA function in the globus pallidus. Brain Research, 116, 353-359.

Roberts, E. (1974). Commentary: $\gamma$-aminobutyric acid and nervous system function-a perspective. Biochemical Pharmacology, 23, 2637-2648.

Tarsy, D., Pycock, C., Meldrum, B., and Marsden, C. D. (1975). Rotational behaviour induced in rats by intranigral picrotoxin. Brain Research, 89, 160 165. 